\title{
Dinámica de coberturas de la tierra en la Subcuenca III de la Cuenca Sur del Lago de Managua, Nicaragua.
}

\section{MSc. Ingrid Ubeda Trujillo}

Investigadora y profesor titular del Departamento de Geografía

Universidad Nacional Autónoma de Nicaragua, Managua (UNAN-Managua)

https://orcid.org/0000-0002-1349-4682

ingrid.ubeda@unan.edu.ni / ingrideut@gmail.com

\section{Dr. Lester Rocha}

Investigador y profesor titular del Departamento de Zootecnia

Universidad Nacional Agraria (UNA)

https://orcid.org/0000-0002-0622-9733

lester.rocha@ci.una.edu.ni

Fecha de recepción: 27 de enero, 2020 / Fecha de aceptación: 21 de marzo, 2020

https://doi.org/10.5377/torreon.v9i25.9857

Palabras clave: cambio de coberturas de la tierra, Cadenas de Markov, regresión logística, método de asignación ordenada.

\section{RESUMEN}

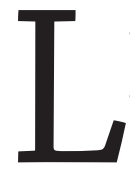

a Subcuenca III de la Cuenca Sur del Lago de Managua, Nicaragua (de ahora en adelante Subcuenca III de Managua) es considerada una zona estratégica para el desarrollo económico y social de Managua, por lo que la dinámica de las coberturas de la tierra que ocurren allí generan conflictos ambientales de diferentes magnitudes. Por lo tanto, la novedad del estudio es la evaluación de la dinámica a través de probabilidades de transición Markovianas haciendo uso de imágenes de satélites Landsat. Además, con base en modelos de regresión logística se determinó las variables biofísicas y socioeconómicas que afectan las transiciones de cada cobertura de la tierra ocurridas en el tiempo. Y adicionalmente, con el método de Asignación Ordenada más los procedimientos anteriores se simuló al año 2025 la dinámica 
de cada cobertura. La capacidad predictiva de los modelos de regresión logística fue evaluada por el método de la curva ROC (Características Operantes de un Receptor). Se consideraron los siguientes períodos: 1997-2003, 2003-2010, 2010-2016, 2016-2025 (simulado). Los resultados indicaron que en el área en estudio durante todos los períodos la agricultura predominó con pocas oscilaciones durante el tiempo. Para el período simulado existen altas probabilidades de que las áreas de bosque y urbano incrementen. Las transiciones estimadas durante los diferentes períodos observados reflejan un alta dinamismo entre las coberturas de la tierra. Durante la simulación, y con pocas excepciones, se observó un dinamismo similar con respecto al último período observado. Las variables biofísicas y socioeconómicas explican las transiciones de cobertura de la tierra en la Subcuenca III de Managua. Sin embargo, la influencia y los cambios de dirección de los efectos no fueron constantes en los períodos evaluados. Adicionalmente, los resultados de la validación indican que el modelo de cambio de coberturas de la tierra fue aceptable.

\section{INTRODUCCIÓN}

La agricultura y la urbanización son los principales motores en la dinámica de la cobertura de la tierra, teniendo un efecto significativo en el paisaje del mundo, y provocando un mayor cambio ambiental en la biosfera (Lambin, Geist y Lepers, 2003; Lambin y Geist, 2006; Lambin, E. F., Meyfroidt, P., Rueda, X., Blackman, A., Borner, J., Cerutti, P. O., Wunder, S, 2014; Han, Yan y Song, 2015). Las investigaciones del modelado de la dinámica de las coberturas de la tierra, se ha centrado en emplear métodos analíticos del proceso estocástico, modelos de regresión logística y espacialmente explícitos, para la evaluación de factores y variables que determinen los cambios en las coberturas de la tierra, y faciliten a su vez explorar su demanda futura (Veldkamp y Lambin, 2001; Overmars, de Koning Veldkamp, 2003; Lambin y Geist, 2006; Verburg, Rousenvell y Veldkamp, 2006; Zhu, Liu, Chen, Zhang y Verburg, 2010; Fuchs, Herold, Verburg y Clevers, 2013; Moulds, Buytaert y Mijic, 2015; Pazúr, Otahel, y Maretta, 2015; Han, Yan y Song, 2015).

La transición de una cobertura de la tierra hacia otras coberturas está influenciado por causas directas e indirectas (Lambin et al., 2003; Lambin y Geist, 2006). Estas causas son diferentes para diversas regiones y escalas (Lambin et al 2003; Zhu et al., 2010). A escala local, las tres variables que influyen en la transición son de tipo socioeconómica, geofísica, y de accesibilidad (Veldkamp y Lambin, 2001; Moulds et al., 2015; Han, et al., 2015).

La variable de tipo socioeconómica, conduce a la expansión agrícola, la construcción de nuevas carreteras y nuevos asentamientos de la población rural (Rudel, T. K., Schneider, M., Uriarte, B. L., Turner, D. R., Lawrence, D., Geoghegan, J. y Grau, R, 2009). La variable geofísica, 
establece que la pendiente y la altitud condicionan la pérdida de bosque mixto para abrir espacios agrícolas (Pineda, Bosque, Gómez, y Plata, 2011).

La variable relacionada con la accesibilidad facilita considerar la teoría original de la localización donde los centros de influencia separan las diversas zonas de usos según los costes de desplazamiento y sobre la base de los factores como los cauces fluviales, fertilidad de los suelos y su producción, transporte y su distribución, núcleos de población, producción del mercado como lo documentó Von Thunen (1826, citado en Alarcón, 2006). En el caso de una cuenca, si tiene acceso a recursos subterráneos, accesibilidad medida en distancias euclidianas, la cobertura de la tierra asignada será agrícola (Henríquez, Azócar y Aguayo, 2006), conllevando a la ampliación de la frontera agrícola, y condicionada a su vez por la infraestructura vial, los asentamientos humanos, y la pérdida de bosque (Pineda et al., 2011). Las coberturas arbustivas reducen paulatinamente su distancia a las ciudades debido al abandono agrícola, conllevando a que los cultivos se encuentren a una distancia media del núcleo poblacional (Peña, 2007). Por otro lado, la cobertura vegetal se reduce totalmente, dando lugar a actividades agropecuarias, habitacionales o industriales (Gong, Juquuan, y Shixiao, 2011; Han, et al., 2015). La selección de variables que determinan los cambios muchas veces dependerá de la disponibilidad de los datos (Lambin, E. F., Turner, B. L., Helmu, J., Geist, H. J., Agbola, S. B., Angelsen, A., Xu, J, 2001).

Las coberturas urbanas, el bosque abierto y cerrado de la Subcuenca III de Managua incrementaron en un $0.98 \%$ y 24.45 \% durante los años 2000 y 2007 debido al manejo del bosque y a la aprobación de proyectos habitacionales (ALMA, 2008). A pesar de contar con esta información, se conoce muy poco sobre la dinámica de cobertura de la tierra en el tiempo y las fuerzas directrices que podrían explicar los cambios. Por lo anterior, este estudio tiene como objetivos evaluar la dinámica de las coberturas de la tierra en la Subcuenca III de Managua, en el período de 1997-2016, y proyectar la dinámica al año 2025, determinar las variables que afectan la dinámica, y evaluar el modelo que determina la dinámica de cobertura de la tierra tanto estadístico como espacialmente.

\section{METODOLOGÍA}

\section{Área de estudio}

La Subcuenca III de Managua se localiza en la Cuenca Sur de Managua, Nicaragua (Figura 1). Tiene una superficie de 17, $337 \mathrm{ha}$, y los municipios que forman parte del área en estudio son: El Crucero, La Concepción, Ticuantepe, Nindirí, y los distritos V y VI de Managua. La Subcuenca III de Managua se extiende desde las costas del Lago de Managua, coincidente con la parte baja del área en estudio (al norte en el mapa), hasta la Meseta de El Crucero, coincidente con la parte alta (al sur en el mapa). 


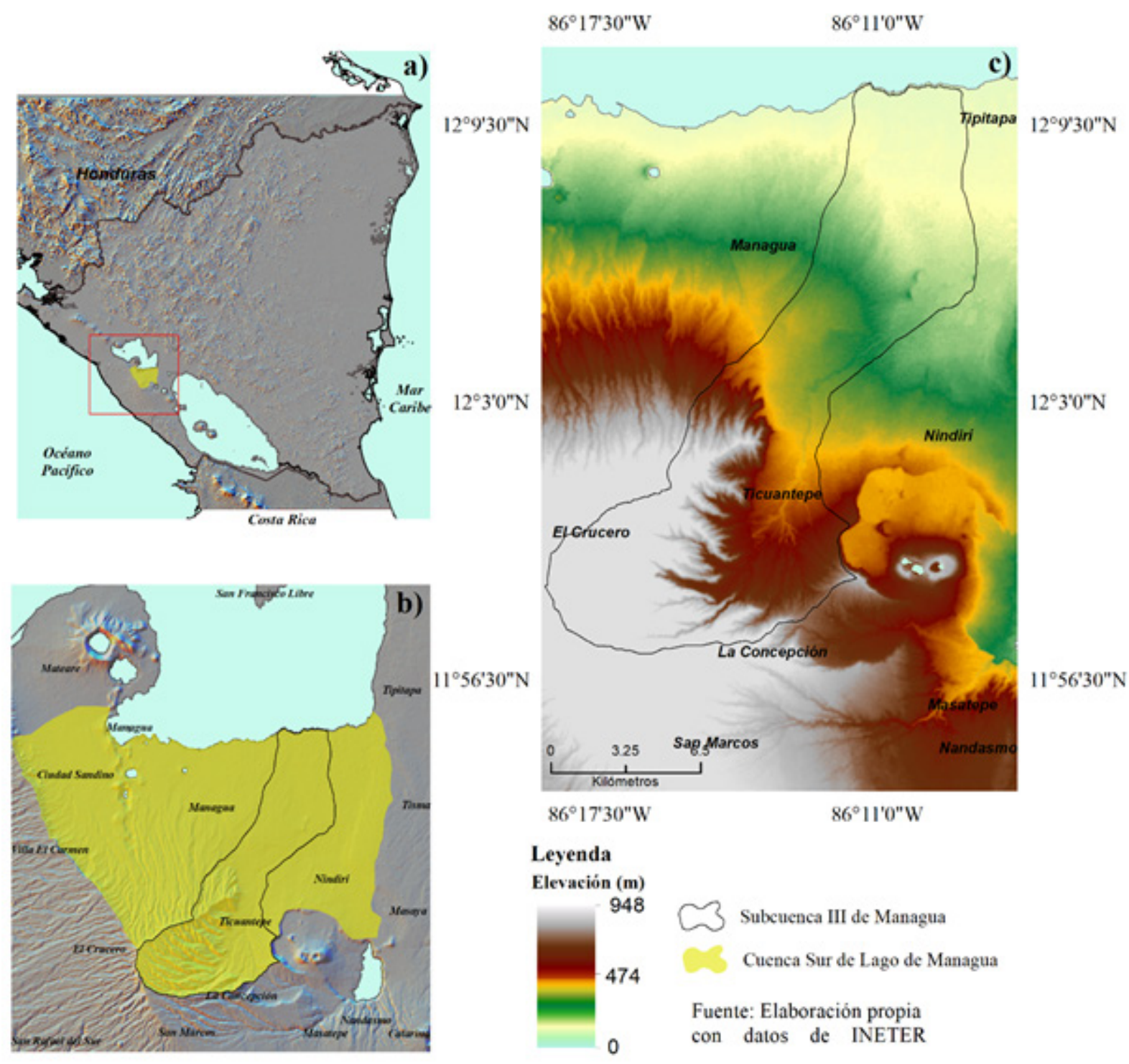

Figura 1. Localización del área en estudio, a) Mapa de Nicaragua, b) Mapa de la Cuenca Sur del Lago de Managua, c) Mapa de la Subcuenca III de Managua.

El total de población ubicada en la Subcuenca III de Managua es de aproximadamente 1, 048,424 (INIDE, 2005). El municipio de Managua (Distrito V y VI) aporta el $79.2 \%$ de la población, Ticuantepe el 10.5 \%, Nindirí 6.5 \%, la Concepción 1.9 \% y el Crucero el 1.9 \% (ALMA, 2008).

La Subcuenca III de Managua presenta una precipitación entre 1700 mm/año y 800 mm/ año. Se caracteriza por altitudes entre 0 a 948 m.s.n.m, con pendientes que oscilan entre $0^{\circ} \mathrm{y}$ $49^{\circ}$, de relieve escarpado a un relieve plano a ligeramente ondulado. También presenta dos zonas de vida, Húmedo Subtropical Premontano y Bosque Seco Tropical (ALMA, 2008). 
La Subcuenca III de Managua es una zona estratégica para el desarrollo económico y social de Managua. En la parte baja del área en estudio se ubican infraestructuras nacionales, industriales y comerciales, así como asentamientos, residenciales y barrios (ALMA, 2008).

\section{MÉTODOS}

\section{Coberturas de la tierra}

A partir de imágenes de satélites Landsat 7 y 8 (sensor ETM+) descargadas del Servicio Geológico de los Estados Unidos (USGS), fueron interpretadas siguiendo el método de Clasificación Interdependiente (FAO, 1996), y segmentadas, con base en la definición de las categorías de coberturas de la tierra: agua, bosque, urbano, agricultura y matorral.

Adicionalmente, se realizó una comprobación de la calidad de los procesos empleados, sobreponiendo la cartografía de las coberturas de la tierra proporcionada por Instituto Nicaragüense de Estudios Territoriales (INETER), las imágenes de satélite de alta resolución (portal Google Earth, el trabajo de reconocimiento en campo, y el conocimiento previo del área en estudio.

Considerando las categorías de coberturas de la tierra definidas previamente y el número de polígonos resultantes en el mapa, se utilizaron 32 puntos control (pixeles de referencia) distribuidos aleatoriamente con un radio de $100 \mathrm{~m}$. Estos puntos de control fueron validados in situ y con base en la imagen de satélite clasificada. La evaluación de la confianza se realizó a partir de una matriz confusión (Chuvieco, 2010) obteniendo una exactitud global del mapa de $81.25 \%$. Seguidamente, se elaboraron los mapas que muestran las categorías de coberturas de la tierra para los años 1997, 2003, 2010, y 2016.

\section{Modelado de la dinámica de coberturas de la tierra}

La dinámica de coberturas de la tierra se evaluó con los métodos de Cadenas de Markov, regresión logística, y Asignación Ordenada (Moulds et al., 2015). Para lo anterior, se determinó las probabilidades de transición de coberturas de la tierra y las variables asociadas al cambio de dichas coberturas tierra.

Con base en los mapas que muestran las categorías de coberturas de la tierra, se cuantificó, para cada transición (1997-2003, 2003-2010, 2010-2016, 2016-2025) y usando "Cadenas de Markov", la probabilidad del cambio de estado de las categorías de coberturas, el cual es un proceso estocástico que satisface la propiedad Markoviana:

$$
\rho\left\{x_{n+1}=j x_{n}=i, x_{n-1} \ldots, x_{1}=i_{1}, x_{0}=i_{0}\right\}=p_{i j}
$$

Para todos los estados $i_{0}, i_{1}, \ldots \ldots, i_{n-1}, i, j$ y todos los $n \geq 0$ 
Lo anterior puede ser interpretado como la distribución condicional de cualquier estado futuro $X_{\mathrm{n}+1}$ dado los estados pasados $X_{0}, X_{1} \ldots \ldots, X_{\mathrm{n}-1}$ y el estado presente es independiente de los estados pasados y depende solamente del estado presente. Ya que las probabilidades son positivas, tenemos que:

$$
\begin{gathered}
P_{i j} \geq 0, \\
i, j \geq 0 \\
\sum_{j=0}^{\infty} P_{i j}=1, i=0,1, \ldots \ldots
\end{gathered}
$$

Las probabilidades de transición se dispusieron en una matriz de probabilidades de estados, en la que la diagonal representa la estabilidad del sistema y los valores cercanos a 1 representa el no cambio. Los valores fuera de la diagonal indican la dinámica de cambio, valores cercanos a 1 representan mayor dinamismo.

\section{Variables asociadas al cambio de coberturas de la tierra}

Para determinar las variables que explican el cambio de coberturas de la tierra para cada período de tiempo evaluado, se consideró que cada cobertura fuera modelada de forma independiente con las variables espacialmente explicitas, aplicando un modelo de regresión logística.

$$
\mathrm{y}=\frac{e^{\alpha+\beta_{x 1}+\beta_{x \ldots .}+\beta_{x 6}}}{1+e^{\alpha+\beta_{x 1}+\beta_{x \ldots}+\beta_{x 6}}}
$$

$X_{1} \ldots \ldots X_{6} \quad$ Variables explicativas.

$\beta_{1} \ldots . . \beta_{6} \quad$ Parámetros de regresión.

Las variables espaciales explicativas del modelado de cambios de cobertura de la tierra fueron: a) Altura, b) distancia a caminos. c) distancia a la ciudad, d) distancia a la red de drenaje, e) pendiente del terreno en grados, y f) cobertura previa de la tierra. En todos los casos, el modelo completo consistió en el ajuste de efectos principales.

Después de ajustar el modelo completo, los términos no significativos fueron removidos paso a paso hasta encontrar el modelo adecuado mínimo. Haciendo uso de los resultados de los modelos se generó un mapa de idoneidad que muestra las probabilidades de ubicación espacial 
de cada cobertura de la tierra en función de cada una de las variables explicativas (Moulds et al., 2015).

La ubicación espacial de las transiciones de cada cobertura de la tierra se realizó mediante el método de Asignación Estocástica Ordenada usando la influencia de las variables explicativas. Este método asignó espacialmente y de forma jerárquica la distribución de las transiciones de cada cobertura creando una matriz de demandas (áreas de coberturas de la tierra) para cada año evaluado y para cada cobertura.

\section{Dinámica futura de coberturas de la tierra al año 2025}

La dinámica de coberturas de la tierra al año 2025 se modeló con base en las transiciones estocásticas de cada cobertura de la tierra para cada período evaluado y en función de las variables espacialmente explicitas que explican cada transición, y que generó el mapa de idoneidad para cada cobertura (Moulds et al., 2015).

\section{Evaluación y validación del modelo de dinámica de coberturas de la tierra}

Con el objetivo de evaluar los resultados derivados del modelo de regresión logística, y atenuar el problema asociado con la autocorrelación espacial que generan los modelos de regresión (Verburg, Soepboer, Veldkamp, Limpiada, y Mastura, 2002; Moulds et al., 2015), se utilizó muestras aleatorias estratificadas de pixeles, y se dividieron los datos en dos grupos: datos para entrenamiento y datos para prueba.

Los datos de entrenamiento fueron utilizados para crear los modelos de regresión, en cambio, los datos de prueba fueron utilizados para evaluar los modelos. Para evaluar la capacidad predictiva de los modelos se utilizó el método de la curva ROC. Este método indica que si el AUC (Área bajo la curva) es igual 1, el ajuste es perfecto, y si el AUC igual a 0.5 el ajuste es puramente aleatorio (Moulds et al., 2015). La validación de la distribución espacial de la transición de cada cobertura se realizó al comparar el mapa simulado de coberturas de la tierra del año 2010 y el mapa obervado para el mismo año (Pontius, Peethambaram, y Castella, 2011).

\section{Análisis de la información}

La evaluación de la dinámica de coberturas de la tierra se realizó con el paquete Land Use Change Modelling (Moulds et al., 2015) del software R (R Core Team, 2018).

\section{RESULTADOS Y DISCUSIÓN}

\section{Coberturas de la tierra durante en el periodo 1997-2025}

En las Figuras 2 y 3 se muestra las coberturas de la tierra: agua, bosque, agricultura, urbano y matorral para los años 1997, 2003, 2010, 2016, y 2025 (simulado). Durante los períodos en estudio se observa que la cobertura agricultura predomina en el área de la Subcuenca III con 
pocas oscilaciones durante el tiempo, a diferencia de la cobertura de bosque y matorral. Por otra parte, se observa una tendencia creciente de la cobertura urbana a través de los años.

En el período 1997-2016, las coberturas de bosque y agricultura disminuyeron en un 10\% y $0.7 \%$, respectivamente, en cambio, se observó un aumento en las coberturas matorral (1.4\%) y urbano (9.5\%). Respecto al período 2016-2025, se prevé un incremento de las coberturas bosque y urbano en un $9 \%$ y $2.5 \%$. Contrariamente, las coberturas agricultura y matorral disminuirán en $3.6 \%$ y $7.8 \%$. Aunque la simulación futura indica la disminución de la cobertura agrícola y el aumento de la cobertura boscosa, el abandono de tierras agrícolas no puede asumirse sin intervención de una política explícita (Rudel, et al., 2009), sin embargo, a medida que los agricultores abandonan los campos menos productivos se genera una probable transición forestal con bosques que se extienden a lo largo de tierras abandonas (Satake y Rudel, 2007).

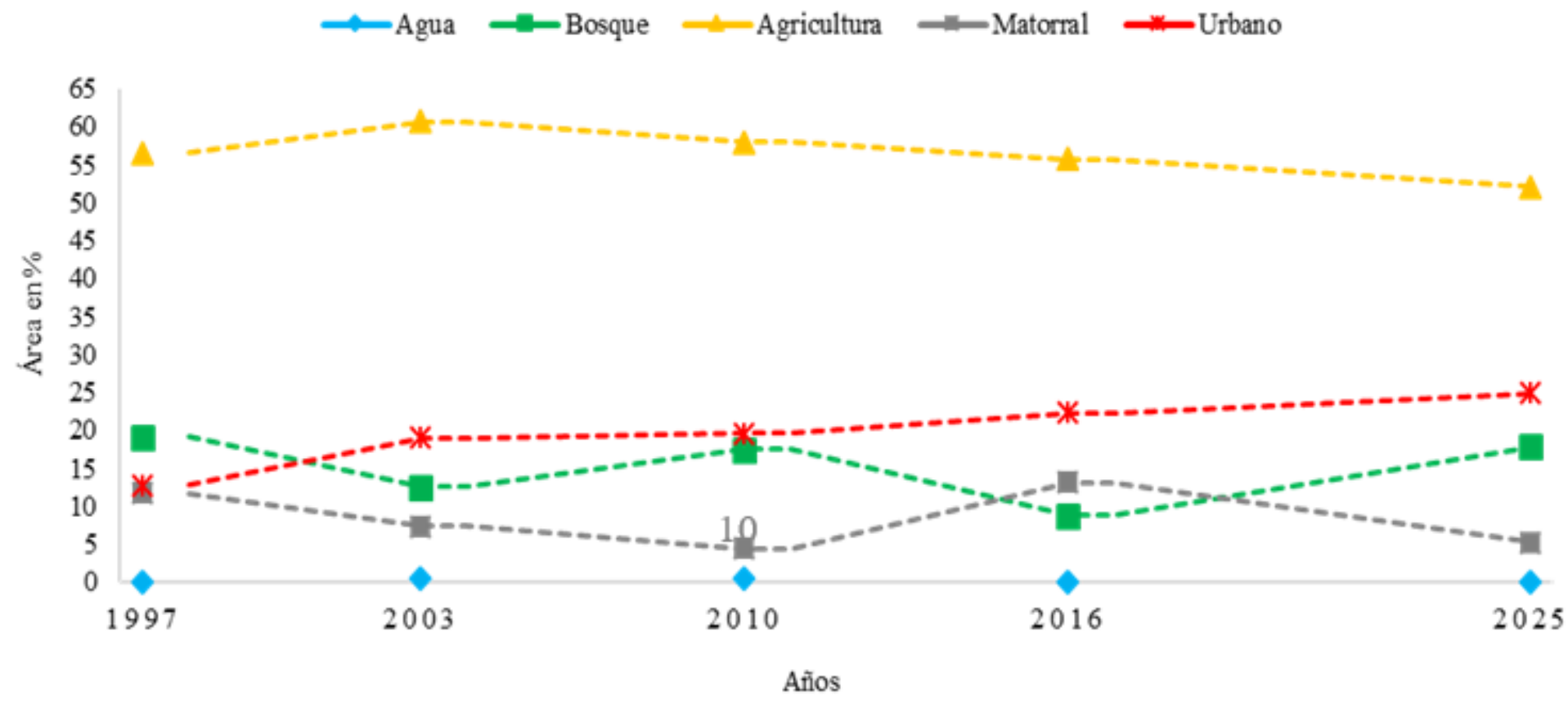

Figura 2. Tendencias anuales de coberturas de la tierra en la Subcuenca III de Managua para los años en estudio. 
$86^{\circ} 15^{\prime} 0^{\prime \prime} \mathrm{W}$

a)

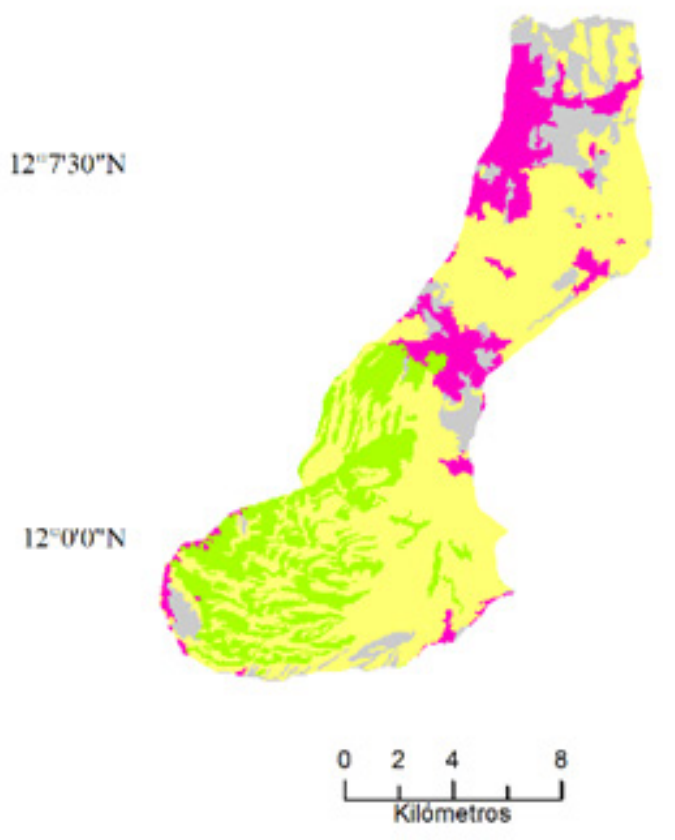

d)

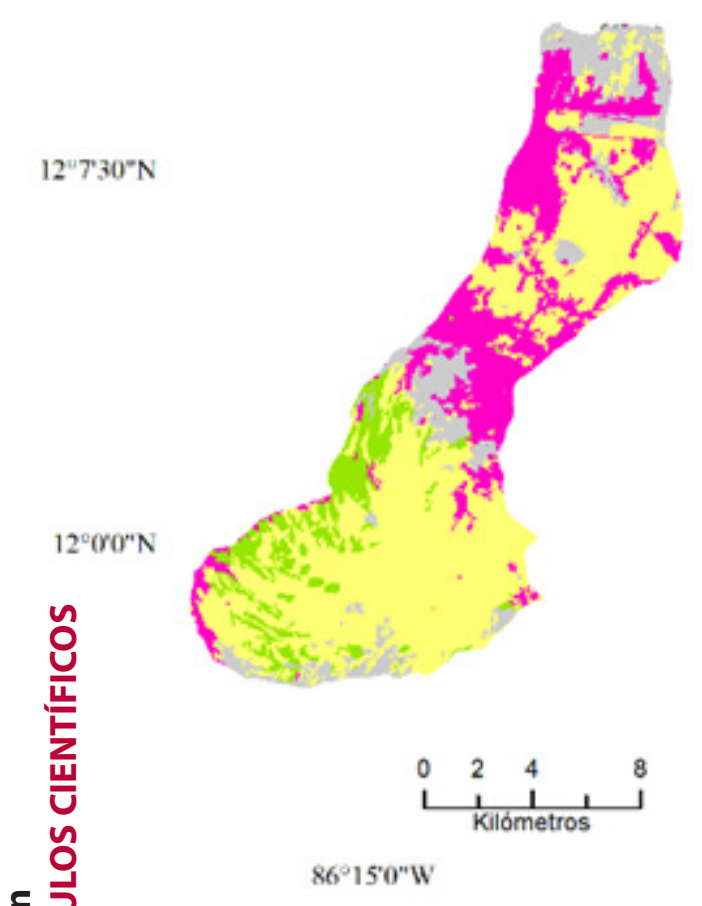

b)
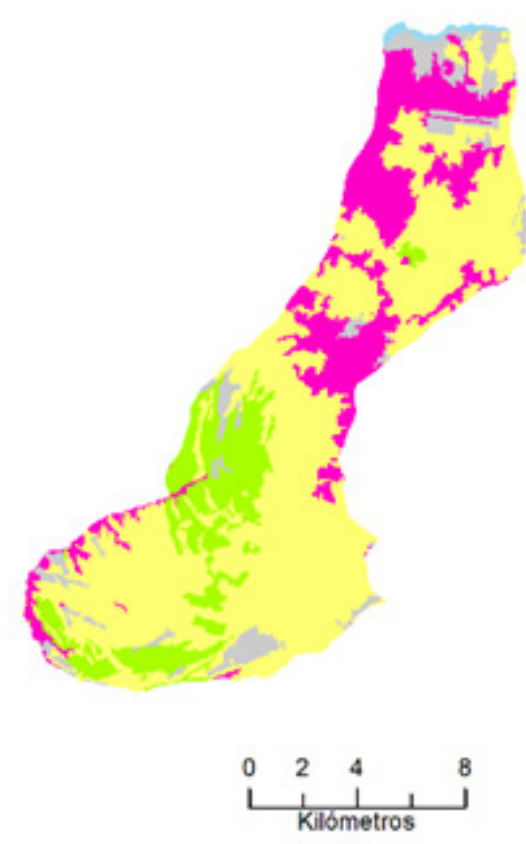

e)

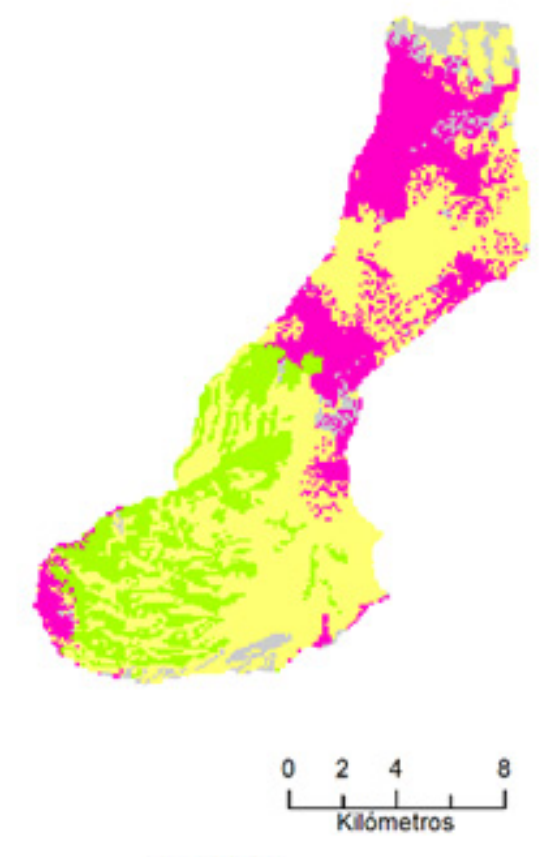

$86^{\circ} 15^{\circ} 0^{\prime \prime W}$ $86^{\circ} 15^{\circ} 0^{\prime \prime} \mathrm{W}$

c)

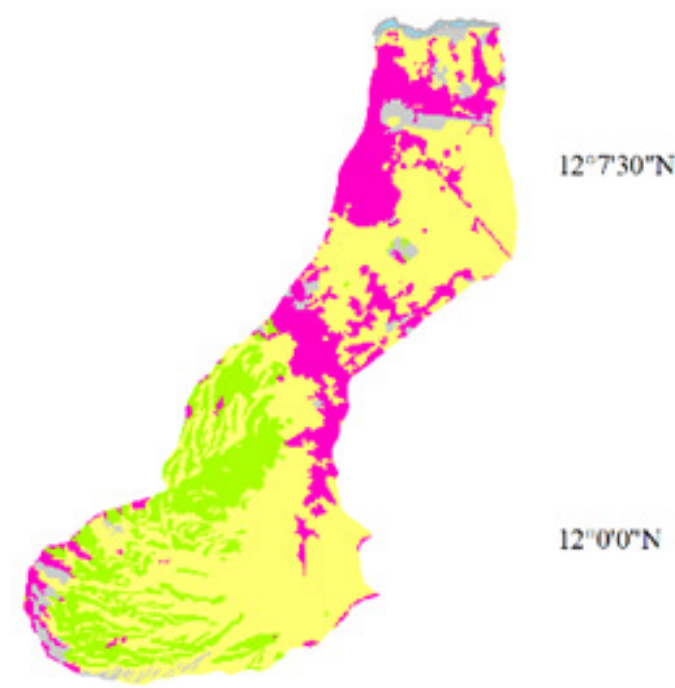

$12^{\circ} 730^{\prime \prime} \mathrm{N}$

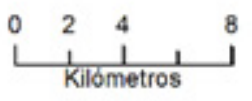

$86^{\prime \prime} 15^{\circ} 0^{\prime \prime} \mathrm{W}$

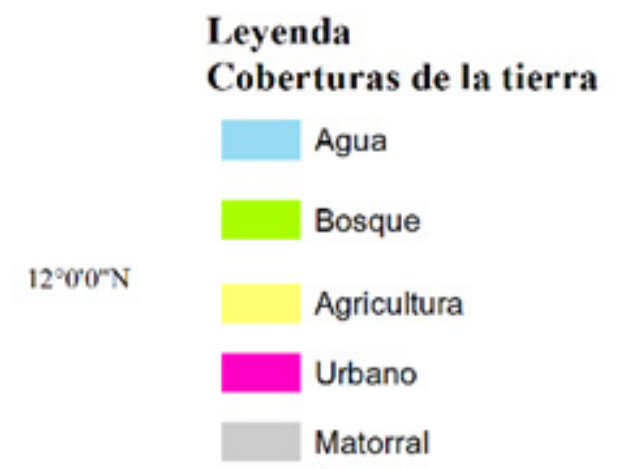

Fuente:Elaboración propia

Figura 3. Coberturas de la tierra en la Subcuenca III de Managua para los años en estudio: a) 1997, b) 2003, c) 2010, d) 2016, e) 2025 


\section{Dinámica de cobertura de la tierra en la Subcuenca III de Managua en el período 1997- 2025}

Las transiciones durante 1997-2003 indican que las categorías de coberturas de la tierra fueron muy dinámicas, siendo agua, agricultura y urbano las que presentaron mayor estabilidad en el período. Las transiciones encontradas con mayores probabilidades de cambio fueron de bosque y matorral hacia agricultura, y de matorral hacia urbano, las demás transiciones presentaron probabilidades bajas o nulas (Cuadro 1). Similar a lo anterior, las coberturas agua, agricultura y urbano fueron las más estables en el período 2003-2010, por otro lado, ocurriendo las transiciones de cobertura con las más altas probabilidades las de matorral y bosque hacia agricultura (Cuadro 2). Diferente a los dos períodos anteriores y con valores medios, durante 2010-2016 las coberturas más estables fueron agricultura y urbano. En este período muy dinámico se observó una probabilidad de matorral hacia agricultura (Cuadro 3).

Entre los períodos observados, el sistema ocurrido entre 2010-2016 fue el que el presentó el mayor dinamismo (valores de la diagonal lejos a 1). Además, se observa como la agricultura juega un rol primario en la dinámica de uso de la tierra en la Subcuenca III. La estabilidad o aumento de la demanda ha sido favorecido por la asignación de créditos a los agricultores, lo que ha permitido el establecimiento de los cultivos de piña y pitahaya, y a la disminución de las áreas de café con sombra y de bosque (ALMA, 2008). Así, las respuestas de las personas a las oportunidades económicas mediadas por factores institucionales impulsan los cambios en la cobertura de la tierra (Lambin et al., 2003; Lambin y Meyfroidt 2010, Zhu, et al., 2010), y es así que la cobertura agricultura se convierte en un agente de deforestación en los países tropicales en desarrollo (Lambin y Geist, 2006).

Por otra parte, la estabilidad o aumento de la demanda de la cobertura urbana en los tres períodos observados es probablemente influenciada por la aprobación de la Ley 677 de Fomento y Acceso a la Vivienda de Interés Social, y por las políticas de vivienda que ha impulsado la Cámara Urbanizadora de Nicaragua. Lo anterior ha facilitado que aproximadamente 180 proyectos urbanos se ubiquen en la parte baja del área en estudio, generando así una densidad promedio de 19 viviendas por hectárea equivalente a 95 habitantes por hectárea (ALMA, 2008).

Durante el período 2016-2025 (simulado, cuadro 4) se prevé la misma tendencia del dinamismo ocurrido en el período 2010-2016, con excepción de coberturas bosque y matorral, las cuales mostraron un aumento y disminución en sus estabilidades, respectivamente.

Por otra parte, se pronostica en el área de estudio un aumento del $30 \%$ más que la población del año 2025 con respecto al año 2005 (ALMA, 2008). El dinamismo que pueda ocurrir en la cobertura urbana durante el período 2016-2025 hace indicar que es posible compensar un aumento poblacional en la Subcuenca III. De este modo la agricultura seguirá siendo una 
actividad central a expensas de una continuada deforestación y las áreas urbanas de Managua seguirán cumpliendo con el principio de servicio de mercado y los municipios que forman parte de la Subcuenca III de Managua serán receptores del crecimiento habitacional debido a la influencia de las políticas vivienda impulsadas en años anteriores. Conclusiones similares enfrentan otras regiones del mundo en el que la política de desarrollo de la ciudad conducirá a la expansión de tierras residenciales y a la concentración de la población (Gong, et al., 2011, Han, et al., 2015).

Cuadro 1. Matriz de probabilidades markovianas para el período de 1997-2003

\begin{tabular}{|c|c|c|c|c|c|c|c|}
\hline & $t_{n+1}$ & \multicolumn{6}{|c|}{2003} \\
\hline & $\mathbf{t}_{\mathbf{n}}$ & $\mathbf{A}$ & B & Ag & $\mathbf{U}$ & $\mathbf{M}$ & $\mathbf{P}_{\mathbf{i +}}$ \\
\hline \multirow{5}{*}{$\hat{\sigma}$} & Agua & 1.000 & 0.000 & 0.000 & 0.000 & 0.000 & 1.000 \\
\hline & Bosque & 0.000 & 0.298 & 0.598 & 0.037 & 0.067 & 1.000 \\
\hline & Agricultura & 0.001 & 0.112 & 0.743 & 0.095 & 0.048 & 1.000 \\
\hline & Urbano & 0.000 & 0.001 & 0.248 & 0.727 & 0.025 & 1.000 \\
\hline & Matorral & 0.041 & 0.045 & 0.345 & 0.315 & 0.254 & 1.000 \\
\hline
\end{tabular}

$A=$ Agua, $B=$ Bosque, Ag= Agricultura, U= Urbano, $M=$ Matorral .

Cuadro 2. Matriz de probabilidades markovianas para el período de 2003-2010

\begin{tabular}{|c|c|c|c|c|c|c|c|}
\hline & $t_{n+1}$ & \multicolumn{6}{|c|}{2010} \\
\hline & $t_{n}$ & $\mathbf{A}$ & B & Ag & $\mathbf{U}$ & $\mathbf{M}$ & $\mathbf{P}_{\mathbf{i}+}$ \\
\hline \multirow{5}{*}{ @) } & Agua & 0.880 & 0.000 & 0.020 & 0.000 & 0.100 & 1.000 \\
\hline & Bosque & 0.000 & 0.557 & 0.393 & 0.013 & 0.038 & 1.000 \\
\hline & Agricultura & 0.000 & 0.141 & 0.731 & 0.101 & 0.027 & 1.000 \\
\hline & Urbano & 0.000 & 0.018 & 0.294 & 0.645 & 0.043 & 1.000 \\
\hline & Matorral & 0.006 & 0.213 & 0.427 & 0.158 & 0.196 & 1.000 \\
\hline
\end{tabular}

$A=$ Agua, $B=$ Bosque, Ag= Agricultura, U= Urbano, $M=$ Matorral . 
Cuadro 3. Matriz de probabilidades markovianas para el período de 2010-2016

\begin{tabular}{|c|c|c|c|c|c|c|c|}
\hline & $t_{n+1}$ & \multicolumn{6}{|c|}{2016} \\
\hline & $\mathbf{t}_{\mathbf{n}}$ & $\mathbf{A}$ & B & Ag & $\mathbf{U}$ & $\mathbf{M}$ & $\mathbf{P}_{\mathbf{i}+}$ \\
\hline \multirow{5}{*}{$\begin{array}{l}\text { 옴 } \\
\text { 유 }\end{array}$} & Agua & 0.039 & 0.000 & 0.022 & 0.013 & 0.926 & 1.000 \\
\hline & Bosque & 0.000 & 0.252 & 0.590 & 0.039 & 0.120 & 1.000 \\
\hline & Agricultura & 0.000 & 0.067 & 0.697 & 0.123 & 0.114 & 1.000 \\
\hline & Urbano & 0.000 & 0.020 & 0.186 & 0.693 & 0.100 & 1.000 \\
\hline & Matorral & 0.000 & 0.038 & 0.336 & 0.180 & 0.446 & 1.000 \\
\hline
\end{tabular}

$A=$ Agua, $B=$ Bosque, Ag= Agricultura, U= Urbano, $M=$ Matorral .

Cuadro 4. Matriz de probabilidades markovianas para el período 2016-2025

\begin{tabular}{|c|c|c|c|c|c|c|c|}
\hline & $t_{n+1}$ & \multicolumn{6}{|c|}{2025} \\
\hline & $\mathbf{t}_{\mathbf{n}}$ & $\mathbf{A}$ & B & $\mathbf{A g}$ & $\mathbf{U}$ & $\mathbf{M}$ & $\mathbf{P}_{\mathbf{i}+}$ \\
\hline \multirow{5}{*}{ 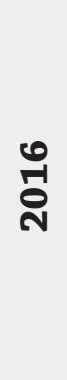 } & Agua & 0.000 & 0.000 & 0.150 & 0.000 & 0.850 & 1.000 \\
\hline & Bosque & 0.000 & 0.511 & 0.462 & 0.018 & 0.008 & 1.000 \\
\hline & Agricultura & 0.000 & 0.210 & 0.634 & 0.129 & 0.027 & 1.000 \\
\hline & Urbano & 0.000 & 0.041 & 0.267 & 0.642 & 0.050 & 1.000 \\
\hline & Matorral & 0.000 & 0.156 & 0.458 & 0.204 & 0.183 & 1.000 \\
\hline
\end{tabular}

A= Agua, $B=$ Bosque, Ag= Agricultura, $U=$ Urbano, $M=$ Matorral .

\section{Efecto de las variables biofísicas y socioeconómicas en la dinámica de la cobertura de la tierra}

Las transiciones de una cobertura de la tierra están condicionadas por el efecto de un complejo de las variables biofísicas y socioeconómicas que varían con el tiempo (cuadros 5 , $6,7,8)$. Durante los períodos evaluados, las transiciones de la cobertura de bosque dependió constantemente de la influencia significativa de las variables altitud, distancia a camino y distancia a la ciudad aunque la dirección del efecto no fue el mismo en el tiempo.

Así por ejemplo, la eliminación de la cobertura de bosque se extendió a sitios con mayor altitud durante los dos primeros períodos evaluados, esto se traduce en una deforestación 
durante esos años pero la situación cambió incrementando dicha cobertura en esas altitudes en último período. Y es que el aumento de la elevación y la pendiente ascendente está relacionada positivamente con la presencia de bosque (Oprsal, Kladivo , y Machar , 2016), aunque la variable altitud condicionan también la pérdida de bosque para abrir espacios agrícolas (Pineda, et al., 2011; Rudel, et al., 2009). Por otra parte, se observó que la influencia y la dirección del efecto de las variables distancia a drenaje, pendiente y cobertura previa de la tierra no fueron constantes durante los diferentes períodos. De esta manera, se observó que las transiciones de la cobertura bosque no fueron afectadas por la distancia a la red de drenaje en el período. Sin embargo, en los dos siguientes períodos la cobertura de bosque incrementó o disminuyó a medida que aumentó la distancia a la red de drenaje.

Las transiciones de la cobertura agricultura pueden ser explicadas por la mayoría de las variables evaluadas las cuales mostraron constancia en la dirección del efecto en todos los períodos. Así, los cambios hacia tierras agrícolas se ven favorecidos hacia mayores altitudes, lejos de los caminos y de los centros urbanos, y en terrenos planos. Así por ejemplo, otros estudios han confirmado que las condiciones topográficas menos favorables son claves para extensión y el abandono de tierra no solo en áreas montañosas marginalmente agrícolas sino también en aquellas con una tradición agrícola intensiva (Oprsal, et al., 2016). Asimismo, la construcción de nuevas carreteras estimulan la expansión agrícola (Pineda, et al., 2011; Rudel, et al., 2009), y la distancia a los centros urbanos en otra variable clave para la ubicación de tierras agricolas (Pazúr, et al., 2015) probablemente debido a la importancia de los costos de transporte en el patrón espacial general de la cobertura de la tierra (Alarcón, 2006). Las transiciones hacia la cobertura urbana estuvo afectada por la mayoría de las variables pero la dirección de los efectos varió en el tiempo. Así, en el primer período evaluado el desarrollo urbano fuera favorecido por la cercanía a la red de drenaje de la Subcuenca III de Managua, sin embargo, durante los siguientes períodos el desarrollo urbanístico se favoreció conforme incrementó la distancia a la red de drenaje y disminuyó la distancia a la ciudad, lo que coincide con lo que sucede en otras regiones (Fuchs et al., 2013). Por otro lado, en las elevaciones más altas, el entorno geográfico limita la expansión de coberturas urbanas aunque la tendencia es el aumento de la demanda de coberturas urbanas en áreas montañosas (Han, et al., 2015).

Al igual que en las otras transiciones, aquellas relacionadas a la cobertura matorral fueron afectadas en diferente medida por el complejo de variables estudiadas y cuyos efectos no fueron constantes a través del tiempo. Con excepción de las variables pendiente del terreno y cobertura previa de la tierra que ejercieron un efecto incremental de la cobertura matorral, las demás variables mostraron efectos bidireccionales en dicha cobertura en los diferentes períodos. 
Cuadro 5. Efectos estimados del modelo de regresión logística para las transiciones de la cobertura bosque

\begin{tabular}{|c|c|c|c|}
\hline \multirow{2}{*}{ Cobertura/ Bosque } & \multicolumn{3}{|c|}{ Periodo de tiempo } \\
\hline & 1997-2003 & 2003-2010 & 2010-2016 \\
\hline Parámetro & Efecto $\operatorname{Pr}(>|z|)$ & Efecto $\operatorname{Pr}(>|z|)$ & Efecto $\operatorname{Pr}(>|\mathrm{z}|)$ \\
\hline Intercepto & - & + & - \\
\hline Altitud & - & - & + \\
\hline Distancia a camino & - & - & + \\
\hline Distancia a la ciudad & + & - & + \\
\hline Distancia a drenaje & ns & + & - \\
\hline Pendiente & + & ns & + \\
\hline Cobertura previa de la tierra & ns & - & - \\
\hline
\end{tabular}

Coeficiente: Efectos: positivo (+) o negativo (-) con una significancia de $P<0.05$, ns: No significativo.

Cuadro 6. Efectos estimados del modelo de regresión logística para las transiciones de la cobertura agricultura

\begin{tabular}{|l|c|c|c|}
\hline \multirow{2}{*}{ Cobertura/ Agricultura } & \multicolumn{3}{|c|}{ Periodo de tiempo } \\
\cline { 2 - 4 } & 1997-2003 & 2003-2010 & 2010-2016 \\
\hline Parámetro & Efecto Pr $(>|z|)$ & Efecto Pr $(>|z|)$ & Efecto Pr $(>|z|)$ \\
\hline Intercepto & ns & - & + \\
\hline Altitud & + & + & + \\
\hline Distancia a camino & + & + & + \\
\hline Distancia a la ciudad & + & + & + \\
\hline Distancia a drenaje & + & + & - \\
\hline Pendiente & - & - & + \\
\hline Cobertura previa de la tierra & ns & + & + \\
\hline
\end{tabular}

Coeficiente: Efectos: positivo (+) o negativo (-) con una significancia de $P<0.05$, ns: No significativo. 
Cuadro 7. Efectos estimados del modelo de regresión logística para las transiciones de la cobertura urbana

\begin{tabular}{|c|c|c|c|}
\hline \multirow{2}{*}{ Cobertura/ Urbana } & \multicolumn{3}{|c|}{ Periodo de tiempo } \\
\hline & 1997-2003 & 2003-2010 & 2010-2016 \\
\hline Parámetro & Efecto $\operatorname{Pr}(>|z|)$ & Efecto $\operatorname{Pr}(>|z|)$ & Efecto $\operatorname{Pr}(>|\mathrm{z}|)$ \\
\hline Intercepto & ns & - & - \\
\hline Altitud & - & + & ns \\
\hline Distancia a camino & - & - & - \\
\hline Distancia a la ciudad & - & - & - \\
\hline Distancia a drenaje & - & + & + \\
\hline Pendiente en grado & - & - & - \\
\hline Cobertura previa de la tierra & ns & + & + \\
\hline
\end{tabular}

Coeficiente: Efectos: positivo (+) o negativo (-) con una significancia de $P<0.05$, ns: No significativo.

Cuadro 8. Efectos estimados del modelo de regresión logística para las transiciones de la cobertura matorral

\begin{tabular}{|l|c|c|c|}
\hline \multirow{2}{*}{ Cobertura/ Matorral } & \multicolumn{3}{|c|}{ Periodo de tiempo } \\
\cline { 2 - 4 } & 1997-2003 & 2003-2010 & 2010-2016 \\
\hline Parámetro & Efecto Pr $(>|z|)$ & Efecto Pr $(>|z|)$ & Efecto Pr $(>|z|)$ \\
\hline Intercepto & ns & ns & - \\
\hline Altitud & + & ns & - \\
\hline Distancia a camino & + & - & + \\
\hline Distancia a la ciudad & - & - & + \\
\hline Distancia a drenaje & - & - & ns \\
\hline Pendiente en grado & + & + & + \\
\hline Cobertura previa de la tierra & ns & + & + \\
\hline
\end{tabular}

Coeficiente: Efectos: positivo (+) o negativo (-) con una significancia de $P<0.05$, ns: No significativo.

\section{Evaluación y validación del modelo de dinámica de coberturas de la tierra}

La evaluación de los resultados derivados del modelo de regresión logística para cada cobertura de la tierra y período de tiempo evaluado, presenta un buen ajuste, reflejado por los 
valores del área bajo la curva (AUC 1). Resultados similares fueron obtenidos por Moulds et al., (2015).

La demanda de cada cobertura es simulada adecuadamente pero, la distribución espacial de transición de cada cobertura no ocurre de esa manera. Así, la demanda real de superficie para la cobertura agricultura era de $56.4 \%$ para el año 2010 y la simulación asignó el 60.5\% para el mismo año observado. Sin embargo, la simulación para la cobertura urbana es aceptable debido a que la diferencia entre la demanda y lo simulado fue de 0 . Contrariamente, la máxima incertidumbre se observa para la cobertura agua, donde la demanda real fue de $0.55 \%$, mientras, en la simulación fue de $0.02 \%$. Por otro lado, la distribución espacial de transición de cada cobertura no es ubicada correctamente en el mapa simulado. Así por ejemplo, algunos sitios de urbanización ubicados en la parte media del mapa de referencia (año 2010) no aparecen en el mapa simulado (año 2010). Para mejorar la simulación espacial es recomendable incluir variables explicativas demográficas y de planificación gubernamental (Pontius, Gil, y Schneider, 2001; Verburg et al., 2002; Gong, et al., 2011).

\section{CONCLUSIONES Y RECOMENDACIONES}

Durante el período evaluado (1997-2025) la agricultura predomina en el área de la Subcuenca III con pocas oscilaciones durante el tiempo. Los resultados para el período simulado (2016-2025) indican un incremento en las áreas de bosque y urbano. Las transiciones estimadas durante los diferentes períodos observados reflejan un alta dinamismo entre las coberturas de la tierra. Durante la simulación, y con pocas excepciones, se observó un dinamismo similar con respecto al último período observado. Además, las variables biofisicas y socioeconómicas explican las transiciones de cobertura de la tierra en la Subcuenca III de Managua. Sin embargo, la influencia y los cambios de dirección de los efectos no fueron constastes en los períodos evaluados. Adicionalmente, los resultados de la validación indican que el modelo de cambio de coberturas de la tierra fue aceptable. La investigación futura estudiará la posibilidad de operacionalizar espacialmente variables de índole sociopolítica que contribuya a la reducción de la incertidumbre y proporcione proyecciones más precisas.

\section{AGRADECIMIENTOS}

A la Universidad Nacional Autónoma de Nicaragua, UNAN-Managua, que a través del Fondo para Proyectos de Investigación (FPI) financió el presente estudio. 


\section{REFERENCIAS}

Alarcón, L. (2006). Factores, predicciones e implicaciones en la asignación de usos de suelo: revisión y reflexiones. Cuadernos de Investigación Geográfica. Cuadernos de Investigación Geográfica, 32, 147-160. https://dialnet.unirioja.es/descarga/ articulo/2283774.pdf

ALMA. (2008). Actualización del estudio de drenaje pluvial de la Subcuenca III de la cuenca sur del lago de Managua. Informe final para la municipalidad de Managua, Alcaldía de Managua, Managua, Nicaragua.

Chuvieco, E. (2010). Teledetección Ambiental (Tercera ed.). Barcelona, España: Ariel.

FAO. (1996). Forest Resource Assessment Programme 1990, Survey of Tropical Forest Cover and Study of Change Processes. (F. a. Nations, Ed.) http://www.fao.org/ docrep/007/w0015e/w0015e00.htm

Fuchs, R., Herold, M., Verburg, P. H., y Clevers, G. (2013). A high-resolution and harmonized model approach for reconstructing and analysing historic land changes in Europe. Biogeosciences, 10(3), 1543-1559. doi:10.5194/bg-101543-2013

Gong, C., Juquuan, C., y Shixiao, Y. (2011). Spatiotemporal dynamics of urban forest conversion through modelurbanization in Shenzhen, China. International Journal of Remote Sensing, 32(24), 9071-9092. do i:10.1080/01431161.2010.549848
Han, H., Yan, C., y Song, J. (2015). Scenario Simulation and the Prediction of Land Use and Land Cover Change in Beijing, China. (Y. D. Wei, Ed.) Sustainability, 7(4), 4260-4279. doi:10.3390/su7044260

Henríquez, C., Azócar, G., yAguayo, M. (2006). Cambio de uso de la tierra y escorrentía superficial: aplicación de un modelo de simulación espacial en Los Ángeles, VIII Región del Biobío, Chile. Geografía Norte Grande(36), 61-74. http://www.redalyc. org/comocitar.oa?id=30003604

Lambin , E. F., Geist, H. J., y Lepers, E. (2003). Dynamics of land-use and landcover change in tropical regions. Annual Review of Environment and Resources, 28(1), 205-241. doi:10.1146/annurev. energy.28.050302.105459

Lambin , E., y Meyfroidt , P. (2010). Land use transitions: Socio-ecological feedback versus socio-economic change. 27(2), 108-118. doi:10.1016/j. landusepol.2009.09.003

Lambin, E. F., y Geist, H. (2006). Land Use and Land Cover Change. Springer-Verlag Berlin Heidelberg. http://www.sysecol2. ethz.ch/AR4_Ch04/Ch4-GreyLit/Al66. pdf

Lambin, E. F., Meyfroidt, P., Rueda, X., Blackman, A., Borner, J., Cerutti, P. O., Wunder, S. (2014). Effectiveness and synergies of policy instruments for land use governance in tropical regions. Global 
Environmental Change, 28, 129-140. doi:10.1016/j.gloenvcha.2014.06.007

Lambin, E. F., Turner, B. L., Helmu, J., Geist, H. J., Agbola, S. B., Angelsen, A., . . X Xu, J. (2001). The causes of land-use and land-cover change:moving beyond the myths. Global Environmental Change(11), 261-269. https://dirzolab.stanford.edu/ wp-content/articles/A_2001/52_2001_ GlobEnvironChange.pdf

Moulds, S., Buytaert, W., y Mijic, A. (2015). An open and extensible framework for spatially explicit land use change modelling: the lulcc $\mathrm{R}$ package. Geoscientific Model Development(8), 3359-3402. doi:10.5194/gmdd-8-33592015

Oprsal , Z., Kladivo , P., y Machar , I. (2016). The role of selected biophosical factors in long-term land-use chance of cultural landscape. Apploed Ecology and Evironmental Research, 14(2), 23-40. doi:doi.org/10.15666/ aeer/1402_023040

Overmars, K. P., de Koning, G. H., y Veldkamp. (2003). Spatial autocorrelation in multi-scale land use models. Ecological Modelling, 257-270. doi:10.1016/S03043800(03)00070-X

Pazúr, R., Otahel, J., y Maretta, M. (2015). The distribution of selected CORINE land cover clases in different natural landscapes in Slovakia: Methodological framework and applications. Moravian
Geographical Reports, 23(1), 45-56. doi:10.1515/mgr-2015-0005

Peña, L. J. (2007). Efectos ecológicos de los cambios de coberturas y usos del suelo en la marina Baixa (Alicante). Tesis para optar al grado de Doctor en Biología, UA, Alicante, España. https://www.pikpotsdam.de/news/public-events/archiv/ alter-net/alumni/tesis-juanpena.pdf

Pineda, J. B., Bosque, J., Gómez, D., y Plata, R. (2011). Analysis of the causative factors behind changes to forest cover in the state of mexico from 1993 to 2000. Boletín de la Asociación de Geógrafos Españoles, 56, 399-402. https://dialnet.unirioja.es/ servlet/articulo?codigo $=3722095$

Pontius, R. G., Peethambaram, S., y Castella, J. C. (2011). Comparison of Three Maps at Multiple Resolutions: A Case Study of Land Change Simulation in Cho Don District, Vietnam. Annals of the Association of American Geographers, 101(1), 45-62. doi:10.1080/00045608.2010.517742

Pontius, R., Gil, J., y Schneider, L. C. (2001). Land-cover change model validation by an ROC method for the Ipswich watershed, Massachusetts, USA. Agriculture, Ecosystems and Environment, 85(1-3). doi:10.1016/S0167-8809(01)00187-6

R Core Team. (2019). R: A language and environment for statistical computing. Viena, Austria: $\mathrm{R}$ Foundation for Statistical Computing. https://www.Rproject.org/. 
Rudel, T. K., Schneider, M., Uriarte, B. L., Turner, D. R., Lawrence, D., Geoghegan, J., . Grau, R. (2009). Agricultural intensification and changes in cultivated areas 1970-2005. Proceedings of the National Academy of Sciences of the United States of America, 110(49), 20675-20680. doi:10.1073pnas.0812540106

Satake , A., y Rudel, T. K. (2007). Modeling the Forest Transition: Forest Scarcity and Ecosystem Service Hypotheses. Ecological Applications, 17(7), 2024-2036. doi:10.1890/07-0283.1

Veldkamp, A., y Lambin, E. (2001). Predicting land-use change. Agriculture, ecosystems and environment, 1-6. doi:10.1016/ S0167-8809(01)00199-2

Verburg, P. H., Rousenvell, D. A., y Veldkamp, A. (2006). Scenario-based studies of future land use in Europe. Agriculture, Ecosystems y Environment, 114(1), 1-6. doi:10.1016/j.agee.2005.11.023
Verburg, P. H., Soepboer, W., Veldkamp, Limpiada, A., y Mastura, S. (2002). Modeling the spatial dynamics of regional land use: the CLUE-S model. Environmental Management, 30(3), 391405. doi:DOI: 10.1007/s00267-0022630-x

Zhu, Z., Liu, L., Chen, Z., Zhang, J., y Verburg, P. H. (2010). Land-use change simulation and assessment of driving factors in the loess hilly region-a case study as Pengyang County. Environmental Monitoring and Assessment, 164(1-4), 133142. doi:10.1007/s10661-009-0880-2 